\title{
Lung ultrasound as diagnostic tool for SARS-CoV-2 infection
}

\author{
Giorgio Bosso ${ }^{1}$ (] - Enrico Allegorico ${ }^{1} \cdot$ Antonio Pagano $^{1} \cdot$ Giovanni Porta $^{1} \cdot$ Claudia Serra $^{1} \cdot$ Valentina Minerva $^{1}$. \\ Valentina Mercurio ${ }^{2}$. Teresa Russo ${ }^{1}$. Concetta Altruda ${ }^{1}$. Paola Arbo ${ }^{1}$. Chiara De Sio ${ }^{1}$. Ferdinando Dello Vicario ${ }^{1}$. \\ Fabio Giuliano Numis ${ }^{1}$
}

Received: 7 May 2020 / Accepted: 16 September 2020 / Published online: 3 October 2020

(c) Società Italiana di Medicina Interna (SIMI) 2020

\begin{abstract}
The aim of this study was to explore the role of lung ultrasound (LUS) in the diagnosis of SARS-CoV-2 infection and to verify its utility in the prediction of lung disease's severity and outcome. Fifty-three consecutive patients presenting to the Emergency Department of Santa Maria delle Grazie Hospital with high suspicion of SARS-CoV-2 infection underwent diagnostic test for SARS-CoV-2 on samples obtained from nasopharyngeal swab as well as complete proper diagnostic work-up that included clinical evaluation, laboratory tests, blood gas analyses, chest CT and LUS. A semiquantitative analysis of B-lines distribution was performed to calculate the LUS score. Patients were divided into two groups according to the results of both SARS-CoV-2 diagnostic test and other exams (Group A = pneumonia due to SARS-CoV2 infection vs Group B = no SARS-CoV2 infection and another definite diagnosis). LUS showed an excellent accuracy in predicting the diagnosis of SARS-CoV-2 infection (area under the ROC curve of 0.92 with a sensibility of $73 \%$ and a specificity of $89 \%$ a the cut-off of 12.5). LUS score was more impaired in SARS-CoV-2 patients $(18.1 \pm 6.0$ vs $7.6 \pm 5.9, p<0.00001)$ and it is significantly negatively correlated with PF ratio values $(r=-0.719, p<0.0001)$. An intrahospital mortality rate of $46 \%$ was found; patients with adverse outcome had significant higher value of LUS, PF, LDH, and APACHE II score. None of these parameters was predictive of mortality. LUS is a useful tool for the early detection of SARS-CoV-2 infection and for the evaluation of the disease severity, but does not predict mortality. Further studies with repeated evaluations of LUS score are needed to further explore the role of LUS in the assessment of severity in SARS-CoV-2 disease and in the monitoring of the response to treatments.
\end{abstract}

Keywords SARS-CoV-2 $\cdot$ Lung ultrasound $\cdot$ LUS score $\cdot$ P/F ratio

\section{Introduction}

By the end of 2019, an outbreak of cases of unusual interstitial pneumonia named Severe Acute Respiratory Syndrome Coronavirus 2 (SARS-CoV-2) rapidly spread from China to the rest of the world [1]. SARS-CoV-2 can present with a wide spectrum of clinical manifestations ranging from flulike syndrome to ARDS with high mortality rate [2]. Initial symptoms such as fever (98\%), cough (76\%), and myalgia or

Giorgio Bosso

giorgio.bosso@aslnapoli2nord.it

1 Department of Emergency Medicine, COVID Care Unit, Santa Maria Delle Grazie Hospital, Via Domitiana, 5, 80078 Pozzuoli, Naples, Italy

2 Department of Translational Medical Sciences, Federico II University, Naples, Italy fatigue (44\%) are common and not specific; therefore, rapid and accurate suspicion of SARS-CoV-2 infection is critical during the outbreak waiting the results of nasopharyngeal swab. In addiction, clinical heterogeneity, the pandemic overcrowding and the difficult to perform an accurate physical examination can lead to underestimate the severity of the disease without the evaluation of the degree of lung involvement [3].

Therefore, lung computed tomography (CT) is considered as the gold standard chest imaging technique to evaluate lung morphology and to perform a quantitative analysis of tissue aeration [4]. However, during outbreak, CT requires a dedicated SARS-CoV-2 radiology and the use of ionizing radiation, precluding a widespread clinical use.

Thus, lung ultrasound (LUS) has been proposed as an alternative imaging technique in Intensive Care Unit [5]. Several studies have explored the role of LUS and its 
correlation with CT scan findings in acute and chronic disease [6]. LUS is a non-invasive, real-time imaging modality; among its advantages, it is easy-to-learn, bedside, repeatable, and free of ionizing radiation. It is considered as an effective tool for early-stage detection of patients with viral pneumonia with an initial normal chest radiography [7]. In addiction, experimental models showed that LUS could allow a semi-quantitative estimation of the extravascular lung water and, indirectly, of the blood oxygenation [8]. The accuracy of LUS in assessing aeration of the lung has been further demonstrated in critically ill patients, both in the context of ventilator-associated pneumonia and acute lung injury through the assessment of LUS score [9].

Growing evidence is showing the usefulness of LUS in SARS-CoV-2 patients from diagnosis to monitoring and follow-up [10].

The aim of this study was to explore the role of LUS in the diagnosis of SARS-CoV-2 infection and to verify if it could be useful in the prediction of lung disease's severity and outcome.

\section{Methods}

\section{Study population}

Consecutive patients admitted to COVID Care Unit of Santa Maria delle Grazie Hospital, Pozzuoli (Naples), for high clinical suspicion of SARS-CoV-2 infection between the 12th of March and the 12th of April 2020 were enrolled. High clinical suspicion of SARS-CoV-2 was defined by the presence of at least two of the following symptoms: fever, dyspnea, cough or desaturation at pulse oximetry. All the patients enrolled were evaluated in a dedicated room and underwent clinical history, physical examination, laboratory tests, arterial blood gas analysis, lung ultrasound, chest $\mathrm{CT}$, and nasopharyngeal swab to obtain the sample for the diagnostic test of SARS-CoV-2 infection through a nucleic acid test by real-time reverse transcription polymerase chain reaction (RT-PCR). In case of negative SARS-Co-V-2 test but persistence of high suspicion of SARS-CoV-2 also at other exams, patients were further tested for SARS-Co-V-2 infection using samples obtained also from bronchoalveolar lavage if needed. According to the results of SARS-CoV-2 test and other diagnostic exams, patients were divided into two groups: Group A (pneumonia due to SARS-CoV2 infection) and Group B (no SARS-CoV2 infection and another diagnosis). Group A patients were admitted to COVID Care Unit and received ventilator support as needed and were treated according to the standard and experimental protocol available [11, 12]; therapies administered and collateral effects were collected. Group B patients were transferred to other wards as needed. Data relative to intrahospital mortality were considered as outcome for the Group A. All participants gave their informed written consent and the protocol was performed in accordance with the principles of the Declaration of Helsinki. The study protocol was approved by the "Campania Centro" Ethical Committee.

\section{Laboratory tests and blood gas analyses}

Peripheral venous blood samples were collected from all individuals for the measurement of cell blood count, glucose, creatinine, AST, ALT, lactate dehydrogenase (LDH), creatinine phosphokinase, fibrinogen, C-reactive protein (CRP), and procalcitonin.

Arterial blood samples were processed and instantly analyzed through a mobile point of care system (Cobas b 123, Roche). The radial artery is the site used for arterial puncture. Oxygenation status is assessed using partial pressure of $\mathrm{O}_{2}\left(\mathrm{pO}_{2}\right)$, partial pressure of $\mathrm{CO}_{2}\left(\mathrm{pCO}_{2}\right)$ and hemoglobin oxygen saturation $\left(\mathrm{SO}_{2}\right)$. The $\mathrm{P} / \mathrm{F}$ ratio was used to compare different values of arterial $\mathrm{pO}_{2}$ in patients receiving different fraction of inspired oxygen $\left(\mathrm{FiO}_{2}\right)$; it was obtained dividing the $\mathrm{pO}_{2}$ by the percent of $\mathrm{FiO}_{2}$ expressed as decimals. Among other parameters, $\mathrm{pH}$ and bicarbonate concentration $\left(\mathrm{HCO}_{3}{ }^{-}\right)$were measured for the evaluation of acid-base disorders; finally, lactate levels were also recorded.

\section{Lung ultrasound}

Lung ultrasound was performed by experts clinicians provided of adequate personal protective equipment with a machine dedicated to SARS-CoV-2 patients. The echo machine was a Samsung HM70A with both linear and convex probes appropriately reset to the study of the lungs. Sonographers were unaware of the results of nasopharyngeal swab. Lung ultrasound was performed bedside in sitting position according to validated protocol described elsewhere [5].

The chest wall was divided into three areas for each side: anterior, lateral and posterior. Each area was further divided into two sections (superior and inferior) for a total of twelve zones of examination (Fig. 1). Each zone was scored according to the lung ultrasound pattern as follows:

- Score 0: presence of A-lines or fewer than three isolated B-lines

- Score 1: presence of multiple well-spaced B-lines

- Score 2: presence of coalescent B-lines with or without small subpleurical consolidations

- Score 3: presence of lung consolidation.

The score observed in each region was recorded and their sum defined the total score (range 0-36). Higher LUS values are indicative of worse ultrasound pattern. 
Fig. 1 Twelve zones' model for LUS calculation. LUS lung ultrasound score

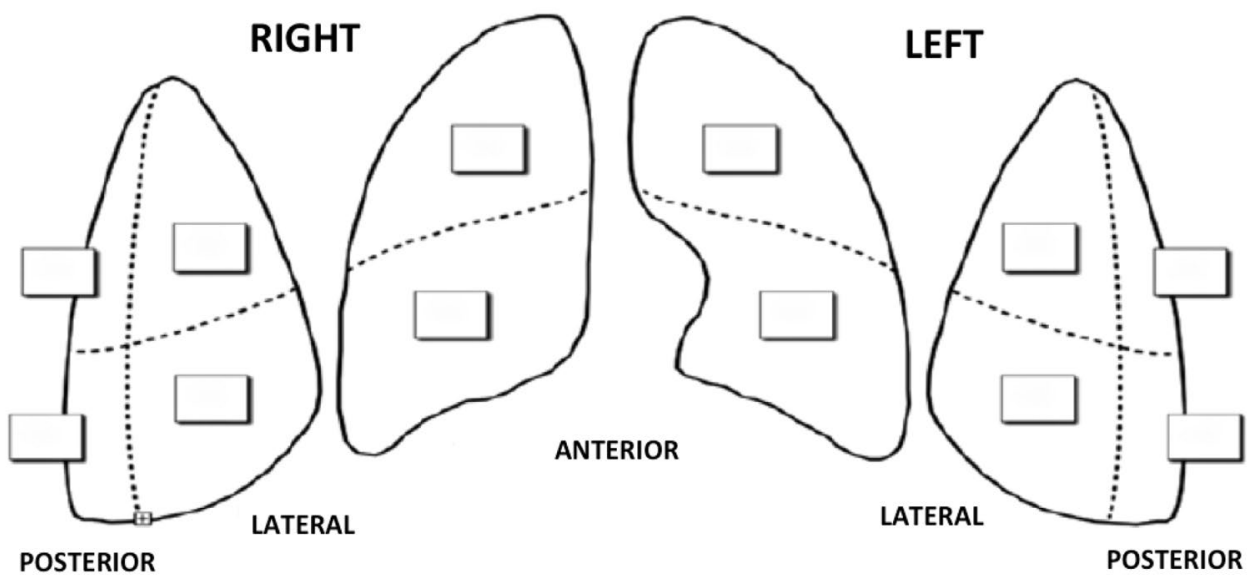

The echographic images were stored according to DICOM standards for subsequent blinded readings by a senior investigator.

\section{Statistical analysis}

Continuous data are expressed as mean \pm standard deviation. Mann-Whitney $U$ test for independent samples was used to compare quantitative variables between groups. Categorical variables were presented as frequencies and percentages and compared using chi-square test with Yates correction. Correlations between variables were examined by determining Pearsons' coefficient. Univariate and multivariate Cox regression analyses was used to determine the hazard ratio (HR) of experiencing the primary endpoint: intrahospital mortality rate was the dependent variable; whereas, LUS, PF, LDH, PCR, PCT and AST were included as independent variables. The ability of LUS to predict the diagnosis was assessed by measurement of the area under the receiveroperating characteristic (ROC) curve (AUC or c-index). The best threshold of the ROC curve was chosen using bootstrap analysis and maximization of the Youden index.

A $p$ value less than 0.05 was considered as statistically significant. All data were collected and entered in an Excel database (Microsoft Office 2016), and statistical analyses were performed using SPSS (IBM SPSS Statistics 25 Version, Inc., Chicago, IL, USA).

\section{Results}

Fifty-three consecutive patients were enrolled in the study. Group A (pneumonia due to SARS-CoV2 infection) consisted of 26 patients, while Group B (no SARS-CoV2 infection and another diagnosis) of 27 patients. Anthropometric characteristics, laboratory tests, blood gas analysis parameters and LUS of the two Groups are summarized in
Table 1. In comparison to Group B, Group A patients had significant higher values of PCR, PCT, LDH and AST with a slight lower count of white blood cells. Regarding the blood gas analysis, SARS-CoV-2 patients presented with a high $\mathrm{pH}$ values with low levels of $\mathrm{pCO}_{2}$ and $\mathrm{pO}_{2}$. According to PF values, 5 patients (20\%) had mild (PF $>300) ; 14$ (54\%) patients had moderate (PF between 200 and 300) and $7(26 \%)$ had severe $(\mathrm{PF}<200)$ hypoxia. Lactate levels were in the normal range without differences between the two Groups.

As showed in Table 1, LUS score was more impaired in Group A patients. SARS-CoV-2 patients presented a typical echo pattern with diffuse coalescent B-lines with small subpleurical consolidations prevalent in the upper lateral and posterior zones of examination. LUS score is significantly negative correlated with PF values in the Group A $(r=-0.719, p<0.0001$, Fig. 2). The interobserver variability for LUS score based on measurements taken in the same individuals by means of Intraclass Coefficient Correlation was excellent (0.96).

Receiver-operating characteristic (ROC) curve analysis demonstrated an excellent ability of LUS in predicting the diagnosis of SARS-CoV-2 infection, with an area under the ROC curve of 0.92 (standard error $0.035, p<0.0001,95 \%$ CI 0.851-0.989) (Fig. 3). Using bootstrap analysis and maximization of the Youden index, the best cut-off was 12.5 (with a sensibility of $73 \%$ and a specificity of $89 \%$ ).

In Group A, 12 patients (46\%) died during the hospitalization with an intrahospital mortality rate of $46 \%$. None of the parameters tested was predictive of mortality (data not shown). Comparison between SARS-CoV-2 patients who survived and who did not survive is shown in Table 2. Patients with adverse outcome had significant higher value of LUS, PF, LDH, and APACHE II score.

In Group B, 5 patients were diagnosed with exacerbation of chronic obstructive pulmonary disease, 5 with septic shock, 6 with sepsis of different origin (3 originated from 
Table 1 Anthropometric characteristics, laboratory parameters and LUS in patients with and without SARS-CoV-2

\begin{tabular}{llll}
\hline & Group A $(n=26)$ & Group B $(n=27)$ & $p$ \\
\hline Sex, M/F & $18 / 8$ & $19 / 8$ & $\mathrm{~ns}$ \\
Age, years & $66 \pm 15$ & $65 \pm 19$ & $\mathrm{~ns}$ \\
APACHE II & $11 \pm 6$ & $11 \pm 6$ & $\mathrm{~ns}$ \\
White blood cell, $\times 10^{3} / \mu \mathrm{L}$ & $8923 \pm 4565$ & $12,396 \pm 5251$ & 0.01 \\
Creatinine, mg/dL & $1.15 \pm 0.45$ & $0.97 \pm 0.36$ & $\mathrm{~ns}$ \\
AST, IU/L & $52.9 \pm 26.4$ & $33.8 \pm 43.1$ & 0.001 \\
ALT, IU/L & $39.3 \pm 25.4$ & $39.2 \pm 43.1$ & $\mathrm{~ns}$ \\
Creatininphosphokinase, IU/L & $531.2 \pm 791.6$ & $211 \pm 335.5$ & $\mathrm{~ns}$ \\
Lactate dehydrogenase, IU/L & $512.1 \pm 258.6$ & $273.6 \pm 85.5$ & $<0.0001$ \\
Fibrinogen, mg/dL & $496.7 \pm 222.4$ & $393.4 \pm 160.6$ & 0.05 \\
C-reactive protein, mg/dL & $15.9 \pm 9.4$ & $4.9 \pm 5.5$ & $<0.0001$ \\
Procalcitonin, ng/mL & $0.49 \pm 0.8$ & $0.23 \pm 0.63$ & $\mathrm{~ns}$ \\
pH & $7.46 \pm 0.03$ & $7.39 \pm 0.08$ & $<0.01$ \\
pCO ${ }_{2}$, mmHg & $32.7 \pm 5.8$ & $38.4 \pm 5.1$ & $<0.01$ \\
P/F & $229 \pm 98$ & $300 \pm 70$ & $<0.01$ \\
Lactate & $1.5 \pm 0.4$ & $2.4 \pm 2.0$ & $\mathrm{~ns}$ \\
LUS score & $18.1 \pm 6.0$ & $7.6 \pm 5.9$ & $<0.00001$ \\
\hline
\end{tabular}

Data expressed as mean \pm standard deviation or absolute number

SARS-CoV-2 severe acute respiratory syndrome coronavirus 2 , LUS lung ultrasound score

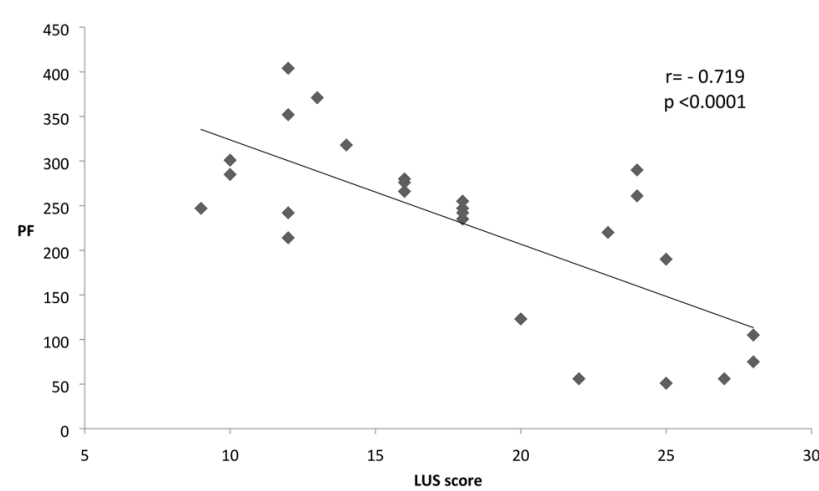

Fig. 2 Correlation between LUS and PF in COVID + patients. LUS lung ultrasound score, $P F$ arterial $\mathrm{pO}_{2}$ to fraction $\mathrm{O}_{2}$ inspired ratio

urinary tract, 2 from cholecystitis, 1 from ab ingestis pneumonia), 6 received a diagnosis of cancer, 5 of acute cardiogenic pulmonary edema. These patients left the COVID Unit after receiving the proper diagnosis and were transferred to general ward, emergency ward, intensive cardiology unit, intensive care unit or surgical ward, according to the specific clinical needs.

\section{Discussion}

We demonstrated an excellent diagnostic accuracy of using LUS score for predicting the diagnosis of SARS-CoV-2 infection in high-suspicion population. In addiction, LUS

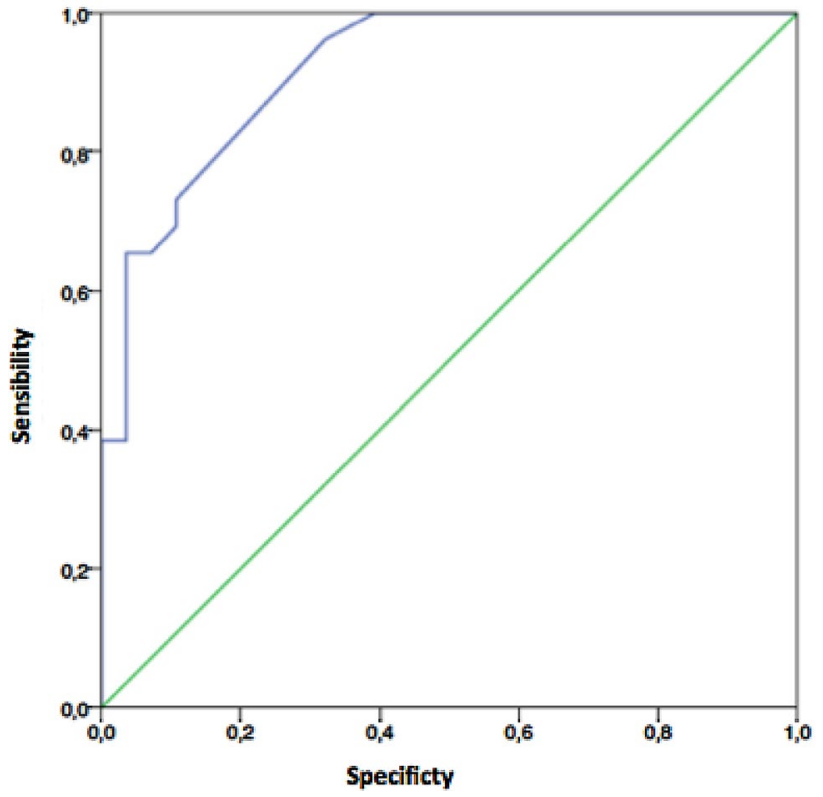

Fig. 3 ROC curve of LUS for diagnosis of COVID+infection. Receiving operating characteristics (ROC) curves of lung ultrasound score (LUS)

score strictly correlates with disease's severity and the degree of hypoxemia assessed through the PF in SARS$\mathrm{CoV}-2$ patients. Some important comments arise.

First, based on LUS, the patients with and without SARSCoV-2 can be separated according to the amount of the LUS score and the echographic characteristics. Even if a 
Table 2 Comparison between SARS-CoV-2 patients divided according to the intrahospital mortality

\begin{tabular}{llll}
\hline & Survived patients $(n=14)$ & Died patients $(n=12)$ & $p$ \\
\hline Sex, M/F & $9 / 5$ & $9 / 3$ & $\mathrm{~ns}$ \\
Age, years & $61.1 \pm 16.5$ & $71.7 \pm 12.3$ & $\mathrm{~ns}$ \\
APACHE II & $7.9 \pm 4.2$ & $14.6 \pm 6.2$ & $<0.01$ \\
White blood cell, $\times 10^{3} / \mu \mathrm{L}$ & $6664 \pm 4765$ & $8176 \pm 3556$ & $\mathrm{~ns}$ \\
AST, IU/L & $43.9 \pm 21.3$ & $63.4 \pm 28.6$ & $\mathrm{~ns}$ \\
Lactate dehydrogenase, IU/L & $376.4 \pm 164.2$ & $670.2 \pm 263.6$ & $<0.01$ \\
C-reactive protein, $\mathrm{mg} / \mathrm{dL}$ & $14.0 \pm 10.7$ & $15.6 \pm 10.7$ & $\mathrm{~ns}$ \\
Procalcitonin, ng/mL & $0.47 \pm 0.9$ & $0.52 \pm 0.7$ & $\mathrm{~ns}$ \\
pH & $7.45 \pm 0.03$ & $7.47 \pm 0.03$ & $\mathrm{~ns}$ \\
pCO ${ }_{2}, \mathrm{mmHg}$ & $34.1 \pm 5.1$ & $32.3 \pm 7.0$ & $\mathrm{~ns}$ \\
P/F & $280.6 \pm 69.3$ & $169.4 \pm 93.4$ & $<0.01$ \\
Lactate & $1.6 \pm 0.5$ & $1.5 \pm 0.4$ & $\mathrm{~ns}$ \\
LUS score & $15.6 \pm 4.5$ & $20.9 \pm 6.5$ & $<0.01$ \\
\hline
\end{tabular}

Data expressed as mean \pm standard deviation or absolute number

SARS-CoV-2 severe acute respiratory syndrome coronavirus 2 , LUS lung ultrasound score pathognomonic sign of SARS-CoV-2 lacks, the most common findings at LUS are the thickening of the pleurae and the appearance of B-lines and lung consolidations while pleural effusion is rarely reported. On further investigation, we found that the LUS score is more increased in anterior and lateral areas, which are non-gravity-dependent regions of lung aeration loss caused by an inflammatory response related to increased lung permeability [13]. From a pathophysiological point of view, changes in the lung parenchyma begin in the distal regions of the lung and progress proximally [14]. These areas that correspond to the 'ground glass' opacities seen on CT scan [15] are already present at the early phases of the disease and can be easily assessed with LUS. This is a possible explanation of the sensibility of LUS to detect the SARS-CoV-2 infection.

Moreover, as showed in the study population, also some laboratory parameters such as the increase of AST, CRP and $\mathrm{LDH}$ and a slight low count of WBC could orient to SARS-CoV-2 diagnosis. These results confirm the findings recently reported by Ferrari et al. [16]. In addiction, regarding the blood gas analysis' results, SARS-CoV-2 patients usually present with a respiratory alkalosis with hypoxia ranging from mild to severe that poorly correspond to the peripheral oxygen saturation, mainly for the left shift of the oxygen-hemoglobin dissociation curve due to alkalosis and low $\mathrm{pCO}_{2}$ levels. For this reason, $\mathrm{P} / \mathrm{F}$ ratio represents the gold standard to evaluate the blood oxygenation in this setting of patients. $\mathrm{P} / \mathrm{F}$ is lower in SARS-Cov-2 patients, particularly those with the worst outcome. It can be considered a global index of tissue aeration [17]. In this regard, it is very remarkable that LUS score has a strong negative correlation with P/F values. LUS score probably identifies the damaged regions of lungs that contribute to hypoxemia through different impaired aeration, vasoconstriction and shunt [14].
Notably lactate levels, that are usually considered a marker of disease severity and prognosis in critically ill patients [18], remain in the normal range also when we divided the SARS-CoV-2 population according to the intrahospital mortality, despite the hypoxia and the increased respiratory rate.

The main strength of our study is that the diagnostic value of LUS has been validated in comparison to a control Group with a similar clinical presentation and in a real-life setting during the outbreak: the echographic findings contribute to correctly direct the patients in the Emergency Department waiting for the results of nasopharyngeal swab.

Some limitations need to be accounted for in this study. First, this was a single-center experience with a relatively small simple size. Furthermore, the present study did not take into account the different treatments for SARS-CoV-19 patients experimented that could highly modify the prognosis and biased the predictive value of basal evaluation of LUS score. Finally, SARS-CoV-2 natural history is not well known, particularly regarding the meaning of its different clinical and radiological phenotype.

In conclusion, LUS is applying not only in the early detection of Sars-CoV-2 infection preceding the nasopharyngeal swab results but also in evaluating the disease severity and monitoring the clinical course. Further studies with repeated evaluations of LUS score can effectively track the course of Sars-CoV-2 disease and its modifications in response to different medical treatment or ventilator support to better characterize its prognostic value.

\section{Compliance with ethical standards}

Conflict of interest None declared. 
Ethical approval The study protocol was approved by the "Campania Centro" Ethical Committee.

Informed consent All the enrolled patients gave their written informed consent to participate in the study.

\section{References}

1. Zhu N, Zhang D, Wang W, Li X, Yang B, Song J, Zhao X, Huang B, Shi W, Lu R, Niu P, Zhan F, Ma X, Wang D, Xu W, Wu G, Gao GF, Tan W (2019) A novel coronavirus from patients with pneumonia in China. N Engl J Med 382:727-733. https://doi. org/10.1056/NEJMoa2001017

2. Ge H, Wang X, Yuan X, Xiao G, Wang C, Deng T, Yuan Q, Xiao X (2020) The epidemiology and clinical information about COVID-19. Eur J Clin Microbiol Infect Dis. https://doi. org/10.1007/s10096-020-03874-z [Epub ahead of print]

3. Lomoro P, Verde F, Zerboni F, Simonetti I, Borghi C, Fachinetti C, Natalizi A, Martegani A (2020) COVID-19 pneumonia manifestations at the admission on chest ultrasound, radiographs, and CT: single-center study and comprehensive radiologic literature review. Eur J Radiol Open 7:100231. https://doi.org/10.1016/j. ejro.2020.100231

4. Li K, Wu J, Wu F, Guo D, Chen L, Fang Z, Li C (2020) The clinical and chest $\mathrm{CT}$ features associated with severe and critical COVID-19 pneumonia. Invest Radiol. https://doi.org/10.1097/ RLI.0000000000000672 [Epub ahead of print]

5. Via G, Storti E, Gulati G, Neri L, Mojoli F, Braschi A (2012) Lung ultrasound in the ICU: from diagnostic instrument to respiratory monitoring tool. Minerva Anestesiol 78:1282-1296 (PMID: 22858877)

6. Man MA, Dantes E, Hancu BD, Bondor CI, Ruscovan A, Parau A, Motoc NS, Marc M (2019) Correlation between transthoracic lung ultrasound score and HRCT features in patients with interstitial lung diseases. J Clin Med 8:1199. https://doi.org/10.3390/jcm80 81199

7. Testa A, Soldati G, Copetti R, Giannuzzi R, Portale G, GentiloniSilveri N (2012) Early recognition of the 2009 pandemic influenza A (H1N1) pneumonia by chest ultrasound. Crit Care 16:R30. https ://doi.org/10.1186/cc11201

8. Zong HF, Guo G, Liu J, Bao LL, Yang CZ (2020) Using lung ultrasound to quantitatively evaluate pulmonary water content. Pediatr Pulmonol 55:729-739. https://doi.org/10.1002/ppul.24635

9. Picano E, Pellikka PA (2016) Ultrasound of extravascular lung water: a new standard for pulmonary congestion. Eur Heart J 37:2097-2104. https://doi.org/10.1093/eurheartj/ehw164

10. Vetrugno L, Bove T, Orso D, Barbariol F, Bassi F, Boero E, Ferrari G, Kong R (2020) Our Italian experience using lung ultrasound for identification, grading and serial follow-up of severity of lung involvement for management of patients with COVID-19. Echocardiography 37:1-3. https://doi.org/10.1111/echo.14664

11. Diurno F, Numis FG, Porta G, Cirillo F, Maddaluno S, Ragozzino A, De Negri P, Di Gennaro C, Pagano A, Allegorico E, Bressy L, Bosso G, Ferrara A, Serra C, Montisci A, D’Amico M, Schiano Lo Morello S, Di Costanzo G, Tucci AG, Marchetti P, Di Vincenzo U, Sorrentino I, Casciotta A, Fusco M, Buonerba C, Berretta M, Ceccarelli M, Nunnari G, Diessa Y, Cicala S, Facchini G (2020) ASL Napoli 2 Nord experience Eculizumab treatment in patients with COVID-19: preliminary results from real life. Eur Rev Med Pharmacol Sci 24:4040-4047. https://doi.org/10.26355/eurre v_202004_20875

12. Pagano A, Porta G, Bosso G, Allegorico E, Serra C, Dello Vicario F, Minerva V, Russo T, Altruda C, Arbo P, Mercurio V, Numis FG (2020) Non-invasive CPAP in mild and moderate ARDS secondary to SARS-CoV-2. Respir Physiol Neurobiol 280:103489. https ://doi.org/10.1016/j.resp.2020.103489

13. Sofia S, Boccatonda A, Montanari M, Spampinato M, D'ardes D, Cocco G, Accogli E, Cipollone F, Schiavone C (2020) Thoracic ultrasound and SARS-COVID-19: a pictorial essay. J Ultrasound. https://doi.org/10.1007/s40477-020-00458-7 [Epub ahead of print]

14. Gattinoni L, Chiumello D, Caironi P, Busana M, Romitti F, Brazzi L, Camporota L (2020) COVID-19 pneumonia: different respiratory treatments for different phenotypes? Intensive Care Med. https://doi.org/10.1007/s00134-020-06033-2 [Epub ahead of print]

15. Hani C, Trieu NH, Saab I, Dangeard S, Bennani S, Chassagnon G, Revel MP (2020) COVID-19 pneumonia: a review of typical CT findings and differential diagnosis. Diagn Interv Imaging. https:// doi.org/10.1016/j.diii.2020.03.014 [Epub ahead of print]

16. Ferrari D, Motta A, Strollo M, Banfi G, Locatelli M (2020) Routine blood tests as a potential diagnostic tool for COVID-19. Clin Chem Lab Med. https://doi.org/10.1515/cclm-2020-0398 [Epub ahead of print]

17. Chiumello D, Mongodi S, Algieri I, Vergani GL, Orlando A, Via G, Crimella F, Cressoni M, Mojoli F (2018) Assessment of lung aeration and recruitment by CT scan and ultrasound in acute respiratory distress syndrome patients. Crit Care Med 46:1761-1768. https://doi.org/10.1097/CCM.0000000000003340

18. Vincent JL, Quintairos E, Silva A, Couto L Jr, Taccone FS (2016) The value of blood lactate kinetics in critically ill patients: a systematic review. Crit Care 20:257. https://doi.org/10.1186/s1305 4-016-1403-5

Publisher's Note Springer Nature remains neutral with regard to jurisdictional claims in published maps and institutional affiliations. 